Memorias del VII Encuentro Nacional de Experiencias en la Enseñanza de la Biología y la Educación Ambiental y II Congreso Nacional de Investigación en la Enseñanza de la Biología

\title{
PROPUESTA DE INVESTIGACIÓN: ANÁLISIS DEL EFECTO DE LAS ZONAS VERDES EN LA CONCENTRACIÓN, AUTOCONTROL Y FUERZA DE VOLUNTAD DE NIÑOS Y NIÑAS
}

\author{
RESEARCH PROPOSAL: AN ANALISIS OF THE IMPACTS FROM GREEN \\ AREAS ON SELF-DISCIPLINE OF CHILDREN
}

\section{Araujo Llamas Roger ${ }^{1}$}

\section{Resumen}

El presente trabajo muestra una propuesta de investigación en el campo de la educación ambiental, que tiene como propósito establecer y comparar el grado de concentración y autocontrol de niños y niñas educados tanto en escuelas "al aire libre" y/o en ambientes rurales como en escuelas tradicionales y/o urbanas. La investigación se realizará implementando entrevistas y aplicando pruebas especializadas reportadas en la literatura que posibilitarán hacer un análisis comparativo e interpretativo de tipo cuantitativo. Vale señalar, que cada vez son más los estudios que señalan al aire libre, la naturaleza y en general las zonas verdes como los ambientes más propicios para un mejor aprendizaje en los niños y niñas, teniendo en cuenta que, en los contextos urbanos los desordenes por déficit de naturaleza van en alza, se ve pertinente realizar estrategias orientadas a la aplicación del modelo "escuela al aire libre" en Hispanoamérica, fortaleciendo este tipo de educación como un modelo eficaz a la hora de superar problemáticas propias de las ciudades y aumentar el rendimiento académico de los estudiantes.

Palabras Clave: Educación al aire libre, concentración, autocontrol. Fuerza de voluntad.

\section{Abstract}

This article aims to demonstrate a research proposal in an environmental educational framework. The purpose of this paper is to establish and compare the capacity of concentration and inhibition of initial impulses and delay gratification of children that study in "Outdoor Education", rural environments, urban contexts or at traditional schools. The research will be conducted by interviews, as well as, by

\footnotetext{
${ }^{1}$ Licenciado en biología Universidad Pedagógica Nacional. Estudiante máster en investigación didáctica de las ciencias experimentales Universidad de Valencia.rogeraraujollamas@gmail.com
} 
Bio-grafia Escritos sobre la Biologia y su Enseñanza.

Edición Extra-Ordinaria. ISSN 2027-1034 P.p 913 - 924

Memorias del VII Encuentro Nacional de Experiencias en la Enseñanza de la Biología y la Educación Ambiental y 11 Congreso Nacional de Investigación en la Enseñanza de la Biología

implementing specialized tests reported in the literature that will enable to conduct a comparative analysis with an interpretive-quantitative approach. It may be noted, that the investigations about learning in nature or in near-home nature shows improvements in the self-discipline and concentration of the students. It is relevant to increase this type of education in Hispano-America as an effective strategy to overcome the typical problems related to the urban context.

Key Words: Outdoor education, self-discipline.

\section{Introducción}

La educación al aire libre, denominada en algunos contextos cómo Forest School, Forest Kindergarden o Outdoor Education ha sido ampliamente utilizada, especialmente en educación infantil en el ámbito anglosajón desde mediados del siglo pasado (O'Brien y Murray, 2006; Burnard, 1998). Por su parte, en Suecia de los años 50's se desarrolló Skoogmulle personaje representativo para la enseñanza de la naturaleza, dirigido a resaltar valores cómo: despertar el interés por la vida al aire libre; disfrutar la esencia de la naturaleza; cuidar la naturaleza; aprovechar los conocimientos provenientes de la naturaleza y aprender todo el año en la naturaleza, fue posible gracias al modelo I Ur och Skur ("Escuelas llueva o haga sol") fundado por Siw Linde en 1985. De la misma manera, modelos similares fueron desarrollándose en diferentes países durante la segunda mitad del siglo pasado, Ella Flautau en Dinamarca (50's) Waldkindergarten en Alemania (60's) Forest Outdoor Schools en Reino Unido (90's) por señalar algunos. Vale mencionar, que existe evidencia qué estos modelos aportan elementos necesarios para desarrollar la concentración, inhibir los impulsos y aumentar el poder de voluntad tanto en niños/as como en adultos (Faber-Taylor, Kuo y Sullivan, 2001).

En España los trabajos realizados por Ayres (2006) y Freire (2011) entre otros, muestran la tendencia global a desarrollar el aprendizaje de la primera infancia en contextos naturales. Es así, como la Fundación Félix Rodríguez de la Fuente e Interprende (entidad fundada en el año 2004) desarrollan "Bosquescuela" una alternativa para que los niños y niñas aprendan en este tipo de contextos. Los principios pedagógicos en los que se basa ésta iniciativa, son punto de referencia para este trabajo, y básicamente son, los tenidos en cuenta en otros países:

1. La naturaleza es el aula.

2. Los niños/as están todo el año al aire libre.

3. La mayor parte del material didáctico proviene del medio natural.

4. El juego libre se considera como el método más natural de aprendizaje. 
Bio-grafia Escritos sobre la Biologia y su Enseñanza.

Edición Extra-Ordinaria. ISSN 2027-1034 P.p 913 - 924

Memorias del VII Encuentro Nacional de Experiencias en la Enseñanza de la Biología y la Educación Ambiental y II Congreso Nacional de Investigación en la Enseñanza de la Biología

5. Los niños/as aprenden mientras viven experiencias reales trabajando sobre contenidos, desarrollando competencias y adquiriendo habilidades.

6. Se carece de instalaciones tradicionales y las sustituye por una cabaña de madera.

7. Dentro de un grupo, los niños/as están mezclados por niveles.

8. Los profesores toman a los niños/as en serio como personas.

Por su parte, en Colombia, La Organización para la Educación y Protección Ambiental (OpEPA) se fundó en 1998 respondiendo a la necesidad creciente de desarrollar este tipo valores en los niños, niñas y jóvenes del país sudamericano, evidenciando la necesidad de implementar este tipo de estrategias en este país.

Para obtener un análisis profundo acerca del papel de las escuelas al aire libre en el aprendizaje de los niños y niñas, el lector puede consultar a Grahn (1997) y Häfner (2003), alemanes que estudian por una parte las diferencias entre niños/as educados bajo este modelo y niños educados bajo modelo tradicional, y por otra el progresivo crecimiento de los Forest Kinder Gardens en este país.

En relación a lo anterior, existen investigaciones como las realizadas por Taylor, Kuo y Sullivan (2001) de la Universidad de Illinois que buscaban obtener información relevante acerca del papel de la naturaleza en la concentración, control de impulsos y fuerza de voluntad en niños/as de 7 a 12 años en contextos urbanos. Vale señalar, que la investigación se realizó en Chicago con población afro descendiente de un inner city (eufemismo utilizado en países anglosajones para hacer referencia a suburbios o barrios pobres dentro de la ciudad), es decir contextos marginados, violentos, con altas tasas de embarazos no deseados en población juvenil, falta de control de impulsos, etc. Los autores pretendían resolver las preguntas: ¿cuál es el papel de la falta de zonas verdes y naturaleza en estos comportamientos? , ¿La falta de estos ambientes puede ser causante del desarrollo de este tipo de actitudes en los niños/as?, concluyeron que la naturaleza próxima al hogar aumenta la autodisciplina (concentración, autocontrol, fuerza de voluntad), además de constatar la importancia de incorporar árboles y hierba en los espacios para niños en las zonas residenciales de las ciudades así como introducir zonas verdes en los patios de las escuelas y tratar de generar una vista lo más verde posible desde las aulas.

En síntesis, la estrategia educativa de Forest Kindergarden surge como una solución viable a lo que Louv (2005) denomina: "Trastorno por Déficit de Naturaleza" generado por el crecimiento y desarrollo de los niños en conglomeraciones urbanas producto de una sociedad altamente industrializada, 
Bio-grafia Escritos sobre la Biologia y su Enseñanza.

Edición Extra-Ordinaria. ISSN 2027-1034 P.p 913 - 924

Memorias del VII Encuentro Nacional de Experiencias en la Enseñanza de la Biología y la Educación Ambiental y 11 Congreso Nacional de Investigación en la Enseñanza de la Biología

dónde los padres mantienen a sus hijos e hijas dentro de casa "sanos y salvos" sin la posibilidad de explorar el medio. En estos niños, la frase "mirar y no tocar" hace parte de su cotidianidad, y esto, sumado al creciente desarrollo tecnológico de su entorno, provoca un contexto no apto para el adecuado desarrollo de habilidades cognitivas y emocionales fundamentales en sus procesos de aprendizaje (Kahn y Kellert, 2002).

Por lo anterior, sería pertinente contrastar la información obtenida en suburbios de grandes ciudades (Ciudad Bolívar, Soacha o Usme, para el caso de Bogotá) con la de escuelas rurales presentes en Sumapaz o Chingaza pudiendo así aportar información necesaria para dilucidar las características y diferencias especificas del contexto colombiano.

Además, es poco lo que se ha indagado sobre éste modelo en otros contextos, y valdría la pena aumentar e investigar estrategias didácticas orientadas a la aplicación del modelo "escuela al aire libre" en Hispanoamérica de una manera contextualizada, para poder así, en primera medida aumentar los conocimientos y valores acerca del ambiente en estos países y por otra parte, poder contrastar los resultados que se obtengan, permitiendo en un futuro evaluar y enriquecer estrategias.

Con base en lo anterior, se refutará o reafirmará la validez de éste exitoso modelo en nuestro contexto, y se dilucidara los matices que se deberán tener en cuenta para optimizar la "escuela al aire libre" en nuestros países. De ésta manera, se espera que la implementación de la estrategia aquí planteada sirva como aporte a una estrategia educativa de larga tradición en numerosos países como Inglaterra (Forest Education Initiative), Escocia (Woods for Learning Education Strategy), Alemania (Waldkindergarten), Suecia (I Ur och Skur, Skogsmulle), Dinamarca (Skov-Skole) y otros como Estado Unidos, Austria, Suiza, Republica Checa, Corea del Sur y Japón, países qué curiosamente obtienen los puntajes en la prueba PISA más destacados. 
Bio-grafia Escritos sobre la Biologia y su Enseñanza.

Edición Extra-Ordinaria. ISSN 2027-1034 P.p 913 - 924

Memorias del VII Encuentro Nacional de Experiencias en la Enseñanza de la Biología y la Educación Ambiental y 11 Congreso Nacional de Investigación en la Enseñanza de la Biología

\section{DISEÑO DE LA INVESTIGACIÓN}

\section{Objetivo}

Establecer y comparar el grado de concentración y autocontrol de niños y niñas educados en escuelas "al aire libre" y/o en ambientes rurales y niños y niñas educados en escuelas tradicionales y/o urbanas de Colombia.

\section{Hipótesis}

Los niños y niñas educados en escuelas "al aire libre" o rurales tienen mayor grado de concentración y autocontrol que los niños y niñas educados en la escuela tradicional o urbanas.

\section{Pregunta-Problema}

Cada vez son más los estudios que señalan al aire libre, la naturaleza y en general las zonas verdes como los ambientes más propicios para un mejor aprendizaje en los niños y niñas. De igual manera, el cada vez más común Desorden por Déficit de Naturaleza (Nature deficit disorder) común en niños/as de contextos urbanos, desarrolla efectos relacionados con déficits de atención, falta de control de impulsos básicos, poca fuerza de voluntad e incluso depresión (Louv, 2005). Además los países que han implementado la educación ambiental y en particular el modelo de escuela al aire libre obtienen puntajes más altos en pruebas que analizan el rendimiento de los estudiantes como el Informe Pisa, lo que podría sugerir que existe un mejor desempeño académico si se educa al aire libre en la infancia, consolidando este tipo de educación como una estrategia eficaz a la hora de superar problemáticas propias de las ciudades.

Por otra parte, la inminente necesidad de desarrollar estrategias educativas encaminadas a la mejora en la comprensión del ambiente por parte de los estudiantes, en mayor medida en los países en vías de desarrollo (donde se evidencia de manera más directa las diversas problemáticas ambientales). Hacen pertinente responder a la pregunta ¿Existe una diferencia en la capacidad de concentración y el grado de autocontrol entre niños y niñas educados en escuelas tradicionales y/o urbanas y escuelas al aire libre o rurales? 
Bio-grafía Escritos sobre La Biología y su Enseñanza.

Edición Extra-Ordinaria. ISSN 2027-1034 P.p 913 - 924

Memorias del VII Encuentro Nacional de Experiencias en la Enseñanza de la Biología y la Educación Ambiental y 11 Congreso Nacional de Investigación en la Enseñanza de la Biología

\section{Metodología}

La metodología que se aplicará es básicamente la reportada por Faber-Taylor, Kuo y Sullivan (2001) en su estudio, con ajustes al contexto local. Se dividirá en tres fases: 1). Lugar y diseño 2). Participantes y proceso 3). Medición y análisis

\section{Lugar y diseño}

El lugar puede ser cualquier localidad o barrio de una ciudad que se encuentre densamente poblado y/o ausente de zonas verdes en buena parte de sus calles, viviendas e Institutos de Educación Distrital en donde sus residentes sean en una proporción representativa niños y niñas entre 5 y 16 años.

Por otra parte, la selección de ambiente rurales o dónde se practique el modelo de escuela "al aire libre" se hará teniendo en cuenta los mismos factores pero en sentido contrario.

\section{Participantes y proceso}

Los entrevistadores deben tener conocimiento previo acerca de cómo realizar entrevistas. Se le deberá suministrar de manera detallada toda la información referente a la investigación a los entrevistados. De la misma manera, tienen el derecho de no responder a cualquiera de las preguntas realizadas y/o de abandonar la entrevista en cualquier momento. De ser posible, se le entregara una pequeña compensación tanto a los adultos como a los niños y niñas que participen en el cuestionario.

Todas las entrevistas y pruebas se realizarán con los participantes acompañados de un adulto responsable. En las pruebas se analizará que tanta naturaleza se puede observar desde el hogar, la concentración del niño/a, el grado de inhibición a impulsos iniciales y el poder de voluntad del participante. Lo anterior, mediante pruebas estandarizadas reportadas en la literatura especializada.

La población objeto de estudio será 200 niños/as entre 7 y 12 años de edad, 100 de ellos pertenecientes a escuelas "al aire libre" o a contextos rurales y 100 a escuelas tradicionales o urbanos. 
Bio-grafia Escritos sobre la Biologia y su Enseñanza.

Edición Extra-Ordinaria. ISSN 2027-1034 P.p 913 - 924

Memorias del VII Encuentro Nacional de Experiencias en la Enseñanza de la Biología y la Educación Ambiental y 11 Congreso Nacional de Investigación en la Enseñanza de la Biología

\section{Medición y análisis}

Se medirá la cercanía del hogar a zonas verdes, y tres tipos de autodisciplina: concentración, inhibición de impulsos (autocontrol) y fuerza de voluntad. El Análisis será comparativo, tomándolo como "un análisis sistemático de observaciones extraídas de dos o más entidades macrosociales -o de varios momentos en la historia de una sociedad- para analizar sus semejanzas y diferencias e indagar sus causas" (Colino, 2007). La medición será de tipo cuantitativo (ANOVA's), separándose los resultados de niñas y niños, ya que se sugiere en investigaciones anteriores que los resultados son significativamente diferentes entre género.

\section{Concentración}

La concentración se medirá por medio de la solución de cuatro tareas por parte de los participantes, estas tareas usualmente se utilizan para la medición de la atención o concentración:

SDMT (Symbol Digit Modalities Test): que básicamente es una evaluación rápida (5 minutos aproximadamente) en dónde el participante realiza una tarea de sustitución múltiple, muy precisa para detección de daño cerebral o cambios en el sistema cognitivo, que nada tienen que ver con los niveles de inteligencia del individuo.

DSB (Digit Span Backwards): es un ejercicio donde el participante tiene que escuchar una secuencia numérica entre 2 y 8 dígitos y luego repetir la secuencia al revés en vos alta, la puntuación se obtiene teniendo en cuenta el número más alto repetido correctamente antes de que se equivoque dos veces consecutivas. $\mathrm{AB}$ (Alphabet Backwards): donde el participante recita el alfabeto al revés comenzando por una letra elegida al azar (por ejp T) dándole la oportunidad de repetir la prueba en tres ocasiones. La puntuación obtenida dependerá del promedio de número de letras recitadas de manera correcta (a la inversa) dividido por el tiempo medio gastado realizando la prueba, es decir la velocidad media con que el participante recita correctamente el alfabeto al revés.

NCPC (Necker Cube Pattern Control): que está diseñado para medir la capacidad para dirigir un esfuerzo mental, este recurso mental se utiliza para gestionar nuestros pensamientos mediante la inhibición de una respuesta con el fin de decir 
Bio-grafia Escritos sobre la Biologia y su Enseñanza.

Edición Extra-Ordinaria. ISSN 2027-1034 P.p 913 - 924

Memorias del VII Encuentro Nacional de Experiencias en la Enseñanza de la Biología y la Educación Ambiental y 11 Congreso Nacional de Investigación en la Enseñanza de la Biología

o hacer algo más. La capacidad para dirigir la atención que nos permite manejar voluntariamente el centro de nuestros pensamientos. Es útil en nuestros esfuerzos para seguir siendo eficaz, productivo, lúcido y atento.

\section{Inhibición De Impulsos}

La inhibición de impulsos se evaluará mediante la combinación de la puntuación de tres tareas en las que, en principio el participante tiende a elegir una respuesta incorrecta, teniendo que discernir la respuesta correcta para lograr obtener un buen rendimiento. Las pruebas son: MFT (Matching Familiar Figures Test); SCWT (Stroop Color-Word Test) y CM (Category Matching).

\section{Fuerza De Voluntad}

Para medir la fuerza o poder de voluntad se utilizará la prueba utilizada por Taylor, Kuo y Sullivan (2001), que a su vez la tomaron de Rodriguez et al (1989), consiste en una prueba que mide la capacidad del niño/a de retrasar recompensas mediante un reto que consiste en preguntarle al participante cual de dos dulces son su favoritos, para luego mostrarle dos bolsas de dichos dulces, una grande y otra pequeña, se le advierte que si espera el tiempo suficiente obtendrá la bolsa de mayor tamaño de lo contrario recibirá la pequeña. El administrador de la prueba luego pide al niño esperar en silencio con los ojos cerrados y sale de la habitación. Los resultados se obtienen midiendo el tiempo total esperado, con un máximo de $15 \mathrm{~min}$.

\section{Análisis Resultados}

Para el adecuado análisis de los resultados se realizarán tablas y gráficos en los que se pueda comparar los datos obtenidos de los dos contextos en las diversas pruebas mencionadas anteriormente. La literatura reporta una estrecha relación entre las áreas verdes y los niveles de concentración, fuerza de voluntad y control de impulsos. De igual manera el análisis muestra una diferencia de género y posibles relaciones entre género y naturaleza, siendo las chicas las que muestran una relación positiva entre concentración y naturaleza. Otras explicaciones alternativas pueden ser tenidas en cuenta, como por ejemplo que, los estudiantes que crecen en contextos con áreas verdes tienen por lo general, padres con mayor cantidad de recursos disponibles para incentivar y desarrollar una mejor autodisciplina. 
Bio-grafía Escritos sobre La Biología y su Enseñanza.

Edición Extra-Ordinaria. ISSN 2027-1034 P.p 913 - 924

Memorias del VII Encuentro Nacional de Experiencias en la Enseñanza de la Biología y la Educación Ambiental y 11 Congreso Nacional de Investigación en la Enseñanza de la Biología

Otra implicación de la investigación puede ir dirigida a reafirmar la importancia de diseñar escuelas que tengan jardines y áreas verdes, siendo estas fundamentales en el desarrollo de la auto-disciplina de los estudiantes.

\section{Agradecimientos}

Al profesor Javier García Gómez y la profesora Amparo Vilches Peña por sus aportes en el Máster de Investigación en Didácticas Específicas de la Universidad de Valencia y a Lisette Johansson por la información proporcionada.

\section{BIBLIOGRAFÍA}

AYRES, A. J. (2006). La integración sensorial y el niño. Sevilla: Editorial Mad S.L. BOSQUESCUELA (s.f). Recuperado el 28 de abril de 2013, de http://bosquescuela.com/quienes-somos/promotores/

BURNARD, S. (1998). Developing children's behaviour in the classroom: a practical guide for Teachers and Students. Falmer Press. p. 77. ISBN 0-75070722-4.

COLINO, C. (2007). "Método comparativo". Diccionario Crítico de Ciencias Sociales. México: Plaza y Valdés.

FABER TAYLOR, A., KUO, F. y SULLIVAN, W. C. (2001). Coping with ADD: The Surprising Connection to Green Play Settings. Environment \& Behavior, 33, 54^77.

FABER TAYLOR, A., WILEY, A., KUO, F. E. y SULLIVAN, W. C. (1998). Growing up in the inner city: Green spaces as places to grow. Environment \& Behavior, 30, $3^{\wedge} 27$.

FABER-TAYLOR, A; KUO, F Y SULLIVAN W. (2001). Views of nature and selfdiscipline: evidence from inner city children. Journal of Environmental Psychology, $21,000^{\wedge} 000$ 
Bio-grafía Escritos sobre La Biología y su Enseñanza.

Edición Extra-Ordinaria. ISSN 2027-1034 P.p 913 - 924

Memorias del VII Encuentro Nacional de Experiencias en la Enseñanza de la Biología y la Educación Ambiental y 11 Congreso Nacional de Investigación en la Enseñanza de la Biología

CÓRDOBA, F. C. (1998.) Fundamentos pedagógicos para la educación Ambiental; Universidad de Córdoba (Colombia). Fondo editorial.

FREIRE, H. (2011): Educar en verde. Ideas para acercar a niños y niñas a la naturaleza. Barcelona: Graó.

GRAHN, P. (1997): Ute pa dagis. Hur anväder barn daghemsgarden? Utformingen av daghems-garden och dess betydelse för lek, motorik och koncentrationsförmaga. Alnarp: Movium.

GRAHN, P., MARTENSSON, F., LINDBLAD, B., NILSSON, P., \& EKMAN, A. (1997) Ute på Dagis Stad and Land 145. Håssleholm, Sweden: Nora Skåne Offset. HÄFNER, P. (2003). Kindergarten in nature and forest in Germany : an alternative to traditional kindergarten in the Pre-school education. Dissertation. The Faculty of Behavioural and Cultural Studies. Institut für Bildungswissenschaft. Germany

JOYCE, R. (2006) Playing Outside Rain or Shine Educational Printing Services Limited. Blackburn ISBN 1-904904-58-0

GONZÁLEZ, G. (2001). Otra lectura a la historia de la educación ambiental en América Latina y el Caribe. Desenvolvimento e Meio Ambiente, n. 3, p. 141-158. Editora da UFPR

MINISTERIO DE EDUCACIÓN NACIONAL (1998). Ciencias naturales educación ambiental, lineamiento curriculares; editorial libros \& libros. Colombia.

ISAÍAS TOBASURA ACUÑA, SEPÚLVEDA GALLEGO. (1997). Proyectos Ambientales Escolares estrategia para la formación ambiental.-1ed-Santa Fe de Bogotá: Cooperativa Editorial Magisterio.

INSTITUTO ALEXANDER VON HUMBOLDT (2010). Educación Ambiental y Biodiversidad, nodo temático del mecanismo de facilitación.

KAHN, P; KELLERT, S. (2002). Children and nature: psychological, sociocultural, and evolutionary investigations. MIT Press. ISBN 0-262-11267-1. 
Bio-grafía Escritos sobre La Biología y su Enseñanza.

Edición Extra-Ordinaria. ISSN 2027-1034 P.p 913 - 924

Memorias del VII Encuentro Nacional de Experiencias en la Enseñanza de la Biología y la Educación Ambiental y 11 Congreso Nacional de Investigación en la Enseñanza de la Biología

KAPLAN, R. (1983). The role of nature in the urban context. In I. Altman \& J. Wohlwill (Eds), Behavior and the Natural Environment, Vol. 6. New York: Plenum Press. pp. $127^{\wedge} 162$.

KAPLAN, R. (1985). Nature at the doorstep: residential satisfaction and the nearby environment. Journal of Architectural and Planning Research, 2, 115^127.

LOUV, R. (2005). Last Child in the Woods: Saving Our Children From NatureDeficit Disorder. Chapel Hill: Algonquin Books.

O'BRIEN, L y MURRAY, R. (2006). A marvellous opportunity for children to learn: a participatory evaluation of Forest School in England and Wales. Forest Research, Farnham. 52 pp. Downloadable from http://www.forestresearch.gov.uk/fr/INFD$\underline{\text { 5Z3JVZ }}$

ORGANIZACIÓN PARA LA EDUCACIÓN Y PROTECCIÓN AMBIENTAL OPEPA (s.f). Líder en educación ambiental en América Latina. Recuperado el 4 de abril de 2013, de http://www.opepa.org/

ORTEGA R. (1997). Manual de Gestión del Medio Ambiente; Editorial Fundación Mapfre.

NOVO, M. (1991). Educación Ambiental; Editorial Rei. Buenos Aires.

RODRIGUEZ, M. L., MISCHEL,W. \& SHODA,Y. (1989). Cognitive Person Variables in the Delay of Gratification of Older Children at Risk. Journal of Personality and Social Psychology, 57, 358^367

SMYTH, J. (1998). The beginning of the end of the beginning. Environmental Conmunicator, Environmental Education NAAEE, v. 28, n. 5, p. 14-16

SHIMIZU, M., TAKAMI, Y., ADACHI, K., OGINO, N. AND TANAKA, H. (2002) a study of the effect of the "forest mulle activity" on environmental learning at schools in Japan. Skogsmullesymposium Journal. Lidingö. 